\title{
Study of physical and chemical assisted physical sputtering of Beryllium in the JET ITER-Like Wall
}

\author{
S. Brezinsek ${ }^{1}$, M.F. Stamp ${ }^{2}$, D. Nishijima ${ }^{3}$, D. Borodin ${ }^{1}$, S. Devaux ${ }^{4}$, K. Krieger ${ }^{4}$, S. \\ Marsen $^{4}$, M. O'Mullane ${ }^{2}$, C. Bjoerkas ${ }^{5}$, A. Kirschner ${ }^{1}$ and JET EFDA contributors ${ }^{\star}$ \\ JET-EFDA, Culham Science Centre, Abingdon, OX14 3DB, UK \\ ${ }^{1}$ Forschungszentrum Jülich, Institut für Energie- und Klimaforschung - Plasmaphysik, \\ 52425 Jülich, Germany \\ ${ }^{2}$ CCFE Fusion Association, Culham Science Centre, Abingdon, OX143DB, UK \\ ${ }^{3}$ Center for Energy Research, University of California at San Diego, 9500 Gilman Dr., La \\ Jolla, CA 92093-0417, USA \\ ${ }^{4}$ Max-Planck-Institut für Plasmaphysik, 85748 Garching, Germany \\ ${ }^{5}$ Department of Physics, University of Helsinki, POB 43, 00014 Helsinki, Finland \\ Corresponding Author: sebastijan.brezinsek@jet.efda.org \\ ${ }^{\star}$ See the Appendix of F. Romanelli et al., Proceedings of the 24th IAEA Fusion Energy Conference 2012, San Diego, USA
}

\begin{abstract}
:
The effective sputtering yield of Be $\left(Y_{B e}^{t o t}\right)$ was determined in-situ by emission spectroscopy of low ionising Be as function of the deuteron impact energy $\left(E_{\text {in }}=25-175 \mathrm{eV}\right)$ and Be surface temperature $\left(T_{\text {surf }}=200^{\circ} \mathrm{C}-520^{\circ} \mathrm{C}\right)$ in limiter discharges carried out in the JET tokamak. Be self sputtering dominates the erosion at high impact energies $\left(E_{i n}>150 \mathrm{eV}\right)$ and causes $Y_{B e}^{\text {tot }}$ far beyond 1. The $Y_{B e}^{\text {tot }}$ drops to low values, below 4.5\%, at the accessible lowest impact energy $\left(E_{i n} \simeq 25 \mathrm{eV}\right)$ achievable in limiter configuration. At medium impact energies, $E_{i n}=75 \mathrm{eV}$, two contributors to the measured $Y_{B e}^{\text {tot }}$ of $9 \%$ were identified: two third of the eroded Be originates from bare physical sputtering $\left(Y_{B e}^{p h y s}\right.$ and one third from chemical assisted physical sputtering $\left(Y_{B e}^{c h e m}\right)$. The later mechanism has been clearly identified by the appearance of $B e D$ A-X emission and quantified in cause of a temperature dependence at which the $B e D$ practically vanishes at highest observed Be limiter temperatures. The recorded $T_{\text {surf }}$ dependence, obtained in a series of 34 identical discharges with ratch-up of $T_{\text {surf }}$ by plasma impact and inertial cooling after the discharge, revealed that the reduction of $B e D$ is correlated with an increase of $D_{2}$ emission. The release mechanism of deuterium in the Be interaction layer is exchanged under otherwise constant recycling flux conditions at the limiter.

The reduction of $Y_{B e}^{\text {chem }}$ with $T_{\text {surf }}$ is also correlated to the reduction of the Be content in the core plasma providing information on the total source strength and Be screening. The chemical assisted physical sputtering, always present at the nominal limiter pre-heating temperature of $T_{\text {surf }}=200^{\circ} \mathrm{C}$, is associated with an additional sputtering channel with respect to ordinary physical sputtering which is surface temperature independent. These JET experiments in limiter configuration are used to benchmark the ERO code and verify ITER first wall erosion prediction. The ERO code overestimates the observed Be sputtering in JET by a factor of about 2.5 which can be transferred to ITER predictions and prolong the expected lifetime of first wall elements.
\end{abstract}




\section{Introduction}

JET is equipped with a first wall material combination comparable to the ITER selection comprising beryllium (Be) in the main chamber and tungsten (W) in the divertor and some recessed wall areas [1]. Be is selected owing to its low atomic number, its low tritium retention and excellent getter properties, but material erosion limits the lifetime of plasma-facing components (PFCs) made of Be. Initial Be erosion modelling of the shaped ITER first wall modules located close to the separatrix [2] predicts high erosion rates and limited armour lifetime [3]. However, uncertainties in the modelling, in particular in the atomic data and sputtering yields, still exist and further benchmark under ITER-comparable tokamak conditions is required. With the present material selection in JET such benchmarks can be performed.

The ITER-Like Wall in JET (JET-ILW) demonstrated successful plasma operation [4],

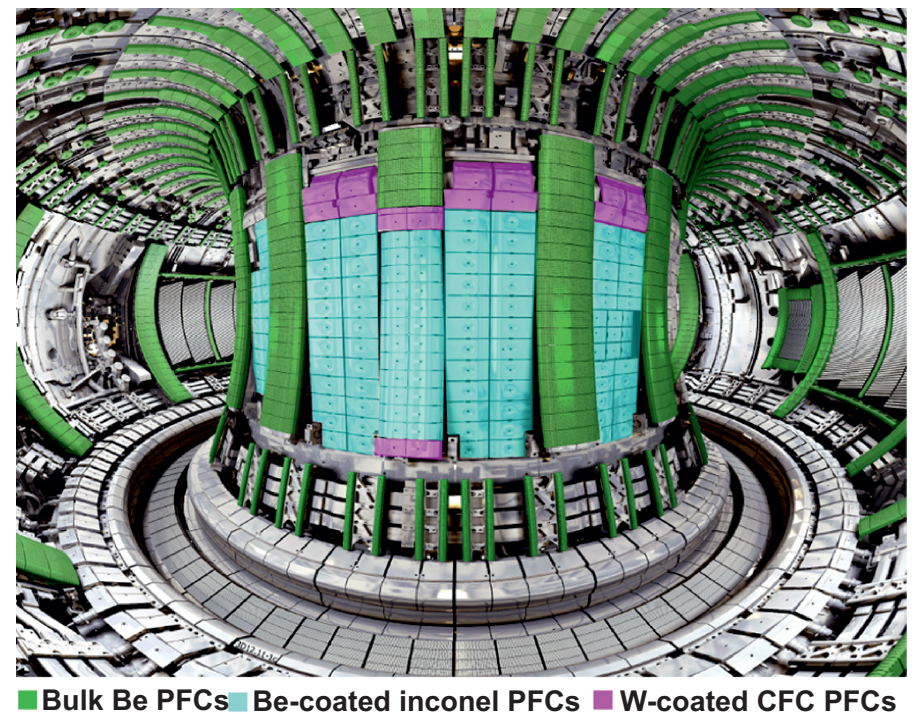

FIG. 1: The JET ITER-Like Wall: Bulk Be (Brush Wellman grade S65J HIP) PFCs are installed at the low and high-field side as main limiter. Be-coated inconcel tiles are used as cladding of the main chamber wall. $W$-coated $C F C$ is introduced at specific areas of high power impact (NBI shinethrough areas, Re-ionisation tiles etc. [8]).

strong reduction of the $\mathrm{C}$ content by a factor 20 , and high plasma purity $\left(Z_{\text {eff }} \simeq 1.2\right)$ in diverted magnetic configuration [5]. The predicted advantages concerning low tritium retention and oxygen gettering were experimentally confirmed by global gas balance studies [6] and optical spectroscopy [7]. All these measurements confirm that Be is the main and dominant intrinsic impurity in limited and diverted plasmas with the JET-ILW. Equipped with its bulk Be limiters (fig 1), the JET-ILW allows dedicated study of Be erosion by optical emission spectroscopy and observation of various transitions of $B e I$ (e.g. $457 \mathrm{~nm}$ ), $B e I I$ (e.g. 527nm) and the $B e D A^{2} \Pi \rightarrow X^{2} \Sigma^{+}$band (band head at $497-500 \mathrm{~nm}$ ) [9] under different plasma conditions and surface temperatures [10]. The total Be sputtering consists of the bare physical sputtering [11] and the chemical assisted physical sputtering $[12]$ - sometimes referred as swift chemical sputtering. However, the composition of the 
total sputtering, its dependence on the impact energy and temperature, the strength of the chemical assisted physical sputtering are not known for a high temperature plasma edge conditions as present in JET-ILW or in future ITER. Experimental input from JET is required to verify the sputtering and transport modelling Monte-Carlo code ERO applied to predict the lifetime of shaped ITER blanket modules [13].

Here, dedicated deuterium plasmas in limiter configuration have been used to vary systematically the local electron temperature $\left(T_{e}\right)$ in the scrape-off layer (SOL), or better, scanning the impact energy of the impinging deuterons $\left(E_{i n}\right)$, as well as, to vary the PFC surface temperature $\left(T_{\text {surf }}\right)$ by plasma impact as they are only inertially cooled. The increase of $T_{\text {surf }}$ is expected to inhibit the sputtering channel via BeD which thermally decomposes at about $540 K$ according to studies in PISCES [14]. Though these are dedicated experiments with variation of one single parameter, impact energy or surface temperature, with the residual plasma and geometry conditions unchanged, the measured Be sputtering yields represent effective yields as averaging of the observation volume, the limiter geometry and the corresponding plasma profile takes place. Nevertheless, clear identification and quantification of both contributors to the Be sputtering yield and their variation with these parameters was achieved.

Details about the experimental set-up and the plasma conditions of the two limiter experiments are given in section 2. The quantification of the two contributors to the Be sputtering yield is presented in section 3 and the variation of the effective yield with the local electron temperature is shown in section 4 . The discussion section 5 deals with the physics mechanisms behind the two processes and discusses accompanied ERO modelling which has been benchmarked with the present data. General remarks concerning the Be source strength in JET in limiter magnetic configuration as well as the degree of Be erosion expected in ITER concludes this contribution.

\section{Experimental set-up and plasma conditions}

Two dedicated experiments in limiter configuration with contact point on the poloidal limiters at the high field side (HFS) (fig. 2a) were carried out in order to study the Be sputtering yield with respect to a) its composition related to chemical and physical sputtering (experiment I), and b) its dependence on the local electron temperature, respectively, the impact energy of the impinging deuterons (experiment II).

\section{$2.1 \quad$ Experiment I}

In experiment I, initially performed for a global gas balance study which is described in more detail in [6], 34 identical deuterium plasma discharges $\left(B_{t}=2.4 T, I_{p}=2.0 \mathrm{MA}, P_{\text {in }}=\right.$ $2.0 \mathrm{MW}$ ) had been executed consecutively with a typical discharge repetition rate of 20min. Typical, global time traces for central electron temperature $T_{e}^{C}$, central density $n_{e}^{C}$, input $P_{i n}$ and radiated power $P_{\text {rad }}$, for the last discharge of the series are depicted in (fig. 2b); the averaging window in the plateau phase of the HFS limited plasmas for analysis is indicated. The discharges showed high reproducibility and though the fuelling rate of deuterium was moderate $\left(\Gamma=6 \times 10^{20} \mathrm{es}^{-1}\right)$ and the radiated fraction was low 
$\left(f_{\text {rad }}=20 \%\right)$, no sign of $\mathrm{W}$ peaking in the core was observed. $Z_{\text {eff }}<1.5$ was determined by the Be content induced by Be sputtering predominantly at the inboard Be limiters as discussed in further detail below. The local plasma conditions at the observation spot
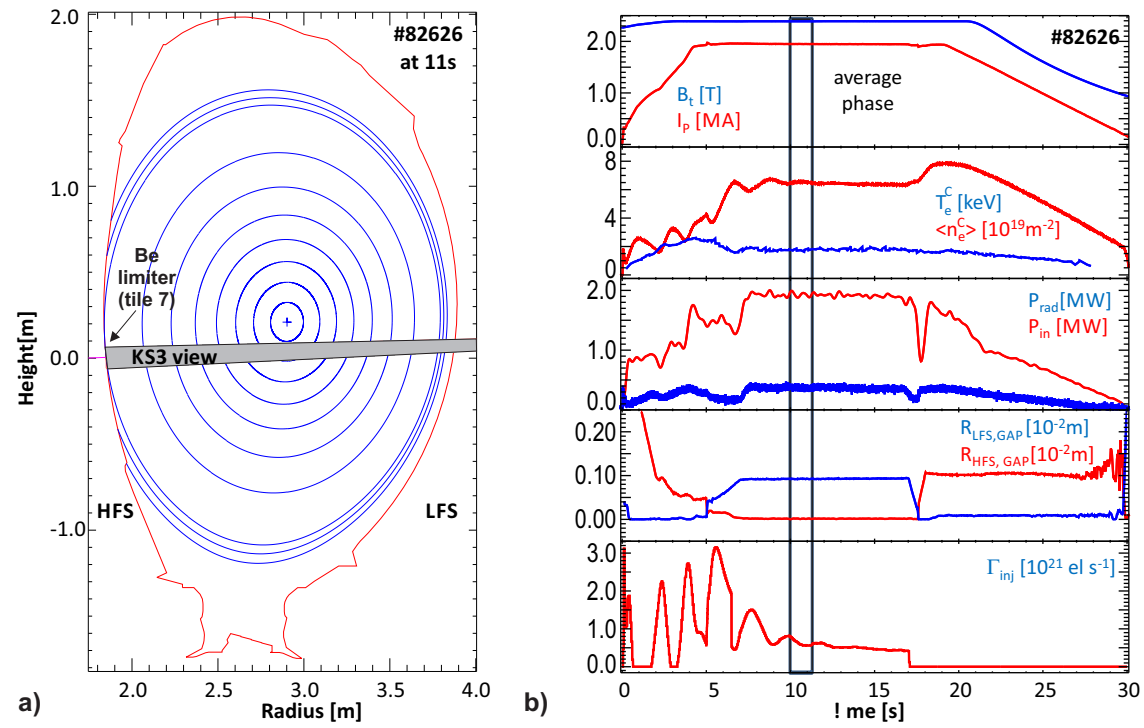

FIG. 2: a) Applied limiter shape in the experiments with the spectroscopic line-of-sight intersecting the inner wall Be limiter tile 7 . b) Evolution of global parameters for the last discharge of experiment (I) [JPN \#82592 - 82626].

of the spectroscopic diagnostic system (Czerny Turner spectrometer, grating: $1200 \mathrm{l} / \mathrm{mm}$, spectral coverage: $420 \mathrm{~nm}-610 \mathrm{~nm}$, spectral resolution: $0.6 \mathrm{~nm}$ FWHM, temporal resolution: $\Delta t=50 \mathrm{~ms}$ ) on the limiter with the line-of-sight depicted in fig. 2a) which lays in the near SOL one tile below the contact point were determined in-situ by line ratio analysis of pairs of BeII lines and low-n deuterium Balmer lines. In fact the local electron temperature was deduced from the line ratio of the $B e I I$ transitions at $467 \mathrm{~nm}$ (transition) and at $436 \mathrm{~nm}$ (transition) which shows a strong dependence on $T_{e}$ in the range of $1-50 \mathrm{eV}$, but is practically independent of $n_{e}$ in the range of $5 \times 10^{17}-1 \times 10^{20} \mathrm{~m}^{-3}$ which covers the conditions present in the JET SOL and edge layer in limiter plasmas. In figure 3a) the calculated line ratio of these two lines according to the ADAS data base [15] is depicted and the actual line ratio measured in experiment I is marked. Figure 3b) shows the corresponding photon flux time evolution during an early and late discharge of the series JPN \#82592 - 82626. Time traces of additional Be and D transitions discussed below are depicted too. All spectroscopic data has been averaged in the time window $t=10 s-11 s$ in the density flat-top phase of the discharge. The BeII line ratio remained constant in the averaged phase of all plasmas executed in experiment I. The local temperature and density deduced from spectroscopy amounts $T_{e}=15 \mathrm{eV}$ and $n_{e}=6 \times 10^{18} \mathrm{~m}^{-3}$ averaged over the observation chord with an area of $0.011 \mathrm{~m}^{2}$ at the limiter location (tile 7 ). These plasma parameters are in agreement with Langmuir probe data from probes embedded in tile 9 which is located poloidally in the same distance away from the plasma contact point but upwards (fig. 2). Assuming electron and ion temperature in the SOL are comparable in the present conditions, the energy of deuterons can be estimated by 

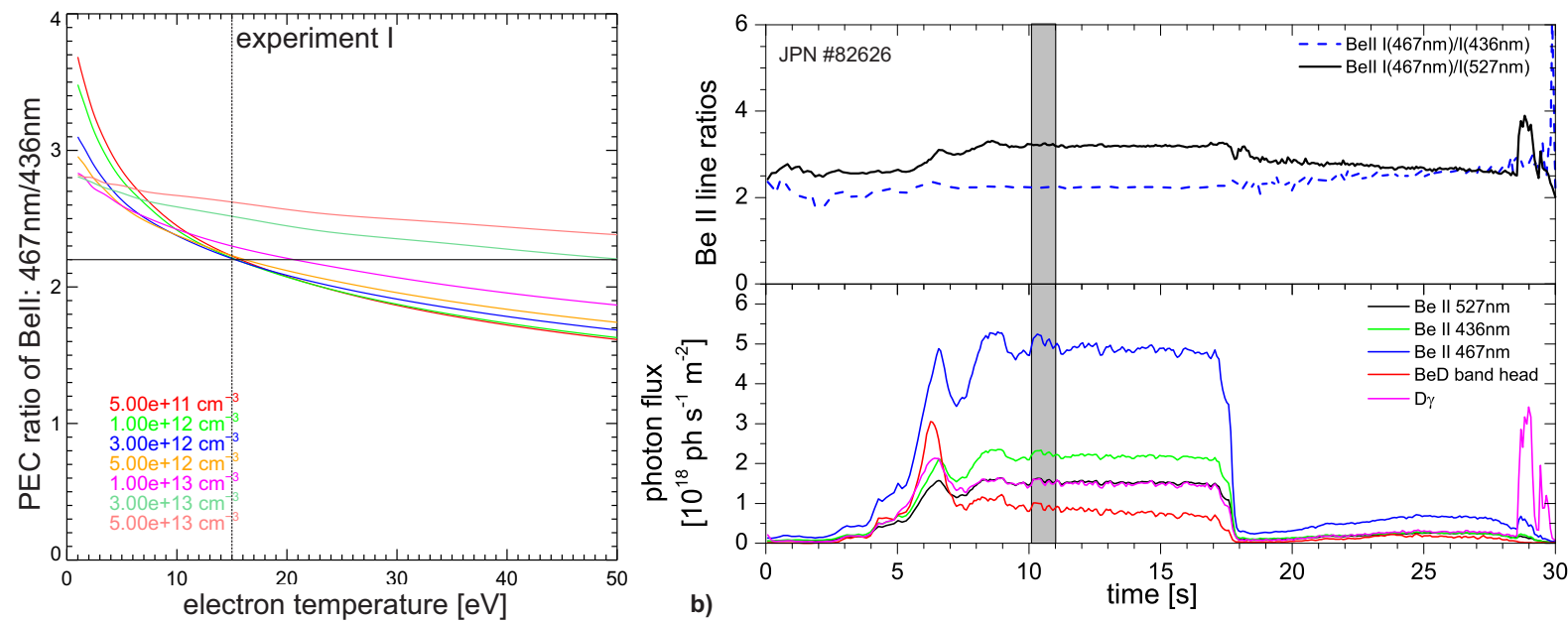

FIG. 3: a) Ratio of photon emission coefficients of BeII for the transitions at $467 \mathrm{~nm}$ and 436nm from ADAS. b) Temporal evolution of three different BeII lines and the corresponding line ratios for discharge JPN 82626.

approximately $E_{i n}=3 k_{b} T_{e}+2 k_{b} T_{i} \simeq 5 \times T_{e}$ [16], therefore, the impact energy of the impinging deuterons can be derived in-situ by the local electron temperature. It shall be pointed out, that $T_{e}=T_{i}$ is a simplification of the relation between $T_{e}$ and $T_{i}$ and most likely only fulfilled at high electron densities where good coupling exist. There is no direct access in JET ohmic limiter plasmas to $T_{i}$, but ohmic limiter plasmas in TEXTOR for similar edge conditions provided for lower electron densities a typical correlation of $1.5 \times T_{e}=T_{i}$ measured in-situ by charge-exchange recombination spectroscopy with a low power diagnostic neutral beam [24]. In the case of JET a similar correlation between $T_{e}$ and $T_{i}$ would lead to a minor increase of the impact energy by $\frac{1}{6}$ for the lower electron density, or better, high temperature cases. This moderate increase of $17 \%$ at the high incident energy end of deuterons will not impact on the general observations made in this contributions.

A peak power flux density of $5 \mathrm{MWm}^{-2}$ is deposited onto the high-field side Be limiter during the flat-top phase of the discharges in experiment I and induces a continuous heat-up of the inertially cooled inner wall limiter tiles during the discharge ( $c f$ Be tile power handling [17]). The temporal evolution of the peak surface temperature measured by IR thermography on the inner wall limiter tile 7 during the second discharge of experiment I is shown in fig. 4a); note that this is a net-erosion zone and no surface layers are present which might affect the interpretation of experimental data. The plasma is limited in the early phase of the discharge $(t=0-5 s)$ to the outer wall limiter. The temperature measured by IR thermography on the inner wall Be limiter tile 7 in this time period reflects therefore the Be bulk temperature, referred as $T_{\text {base }}$ below, which is almost in thermal equilibrium at the beginning of the discharge as confirmed by thermo-couples. The plasma discharges in experiment I were executed with a typical repetition rate of 20min; fig. 4b) shows the discharge-to-discharge evolution of $T_{\text {base }}$ determined by the IR thermography when it was technically available. As it can be seen each plasma pulse in experiment I ratchets up the bulk limiter temperature (JPN \#82592 - 82601) until 


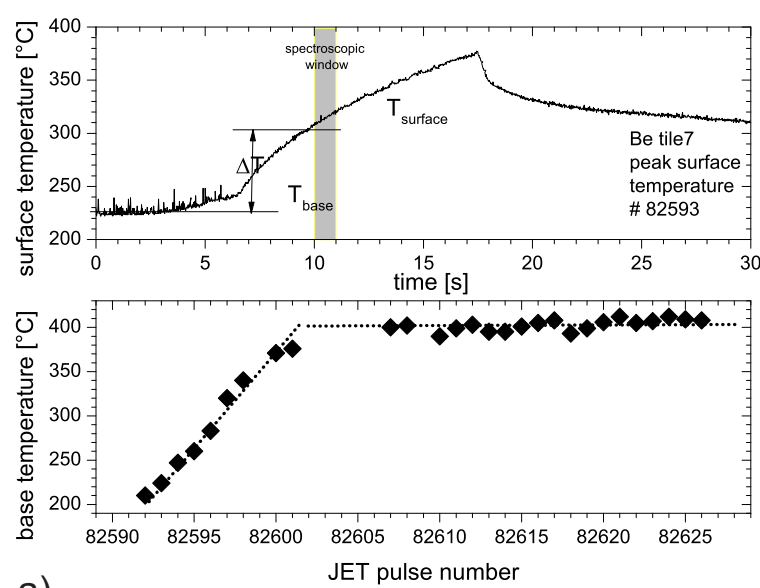

a)

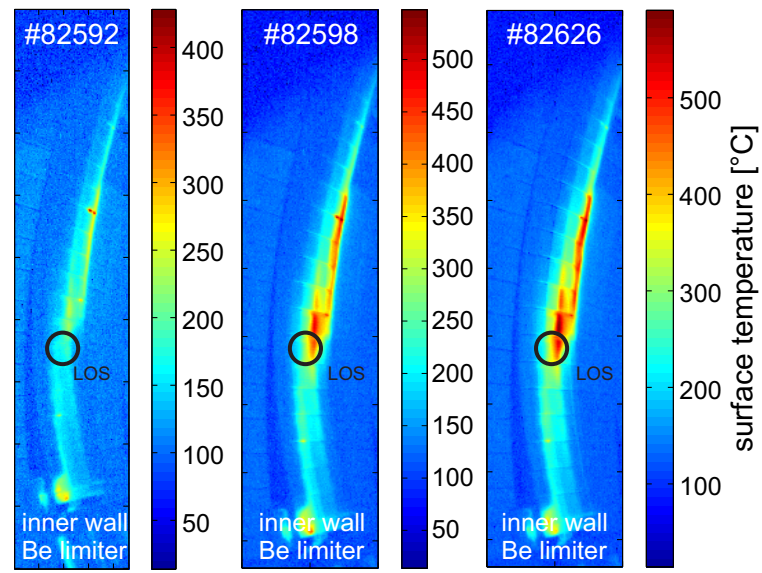

b)

FIG. 4: a) Top: Temperature evolution of the observed Be tile in one discharge. Bottom: Temperature evolution of the observed Be tile in all discharges of experiment (i). b) $2 D$ distribution of the temperature on the inner wall Be limiters for three dedicated discharges of the series of 34 identical plasmas in experiment (i) [JPN \#82592 - 82626].

equilibrium is reached between heating and cooling (JPN \#82602 - 82626). Because of all plasma parameters remain constant in the series of discharges, experiment I provides a fine scan of the base temperature at the beginning of the discharge in the range between $200^{\circ} \mathrm{C}$ and $420^{\circ} \mathrm{C}$ of inner wall Be limiter tile 7 which is in view of the spectroscopic system (line-of-sight in fig. 2a). The lower temperature limit is determined by the active heating of the JET first wall to $200^{\circ} \mathrm{C}$. The actual peak surface temperature $T_{\text {surf }}$ during the plasma excursion rises during the discharge (fig. 4a)) adding typically $\Delta T$ of about $80 K-120 K$ at the sampling point $(t=10-11 s)$ used for the optical spectroscopy; the maximum value of the surface temperature lays even higher at about $150 \mathrm{~K}$ above the base temperature. It should be noted that this $\Delta T$ refers only to the peak value within the tile 7 surface from a complex temperature distribution as depicted in fig. 4c) in the full 2D picture. More information about the power deposition pattern, the associated power decay lengths, shadowing effects of neighboured limiters and temperature measurement uncertainties of the IR system can be found in [26]. As the optical spectroscopy observation chord includes a larger area than the peak spot, averaging takes place and we refer in the following to $T_{\text {base }}$ as reference temperature which is closer to the actual averaged temperature of tile 7 . However, the averaged surface temperature range between $T_{\text {surf }}=200^{\circ} \mathrm{C}$, for the initial temperature value of the first discharge, and $T_{\text {surf }}=520^{\circ} \mathrm{C}$, for the temperature at the sampling time point in the first discharge of experiment I where thermal equilibrium is reached, is covered in the analysis and used in the final discussion in section 5 . 


\subsection{Experiment II}

Experiment II aims in the study of the Be sputtering as function of the impact energy $E_{i n}$ of the deuterons, respectively, of the local temperature $T_{e}$ and density $n_{e}$ at the contact point under comparable limiter temperature conditions. Seven ohmic and highfield-side limited discharges $\left(B_{t}=2.8 T, I_{p}=2.0 \mathrm{MA}\right)$, timely separated in two bunches of discharges at different days, have been performed varying $T_{e}^{C}=1.7-3.4 \mathrm{keV}$ and $n_{e}^{C}=2.7-7.0 \times 10^{19} \mathrm{~m}^{-3}$ by solely deuterium fuelling. The global time traces of the discharges with lowest and highest fuelling are depicted in fig. 5 showing the large variation in the density and simultaneously to an almost reciprocal plasma temperature change. The low fuelled discharges with the highest edge electron temperatures reach high Be impurity concentrations with $Z_{\text {eff }}$ in the hottest plasma edge case (JPN \#80319) of about 4.0 which indicates that strong Be self sputtering must take place leading to a Be-rich plasma. The corresponding high fuelling case (JPN \#80323) reaches in contrast $Z_{\text {eff }}=1.2$ which is close to values typical obtained in divertor conditions with an impurity content of about $1.0 \%$ Be. The full inner-wall limited time window period between $t=7 \mathrm{~s}$ and $t=12 \mathrm{~s}$
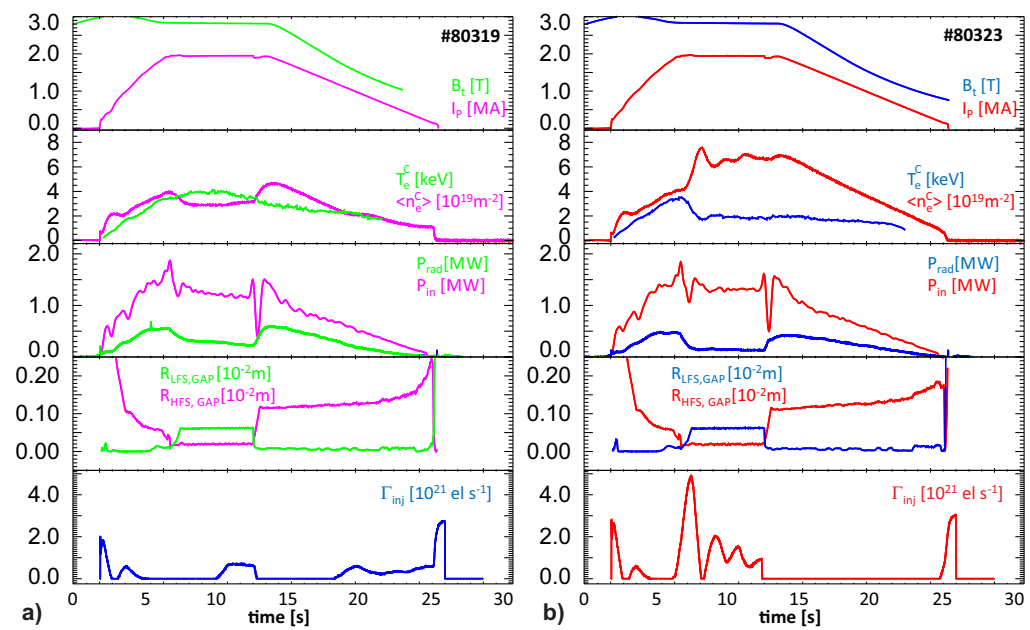

FIG. 5: Timetraces of global plasma parameters of discharges in limiter configuration at lowest (a: JPN \#80319) and highest fuelling level (b: JPN \#80323) used to vary the impact energy $E_{\text {in }}$ of deuterons at the limiter contact point.

was used for the analysis. The corresponding local plasma conditions were determined in-situ by BeII spectroscopy averaged over the line-of-sight on the limiter applying the before described line-ratio technique.

The time between two individual discharges in one subset of discharges as well as the small number of consecutive discharges in limiter configuration in this experiment minimised a ratch-up of the limiter temperature from discharge to discharge. $T_{\text {surf }}$ is in this case close to $T_{\text {base }}$, and both are close to the bulk limiter temperature of $200^{\circ} \mathrm{C}$ achieved by active heating. The corresponding effective sputtering values averaged concerning the area of the line-of-sight on the limiter are therefore representative of the standard condition case; temperature variation from discharge to discharge is not considered in the analysis of this experiment. Initial results on the Be sputtering yield in connection with ERO modelling 
from a subset of data have been presented in [8].

\section{Contributors to the total Be sputtering yield}

\subsection{Interpretation of spectroscopic Be and D-containing emis- sion}
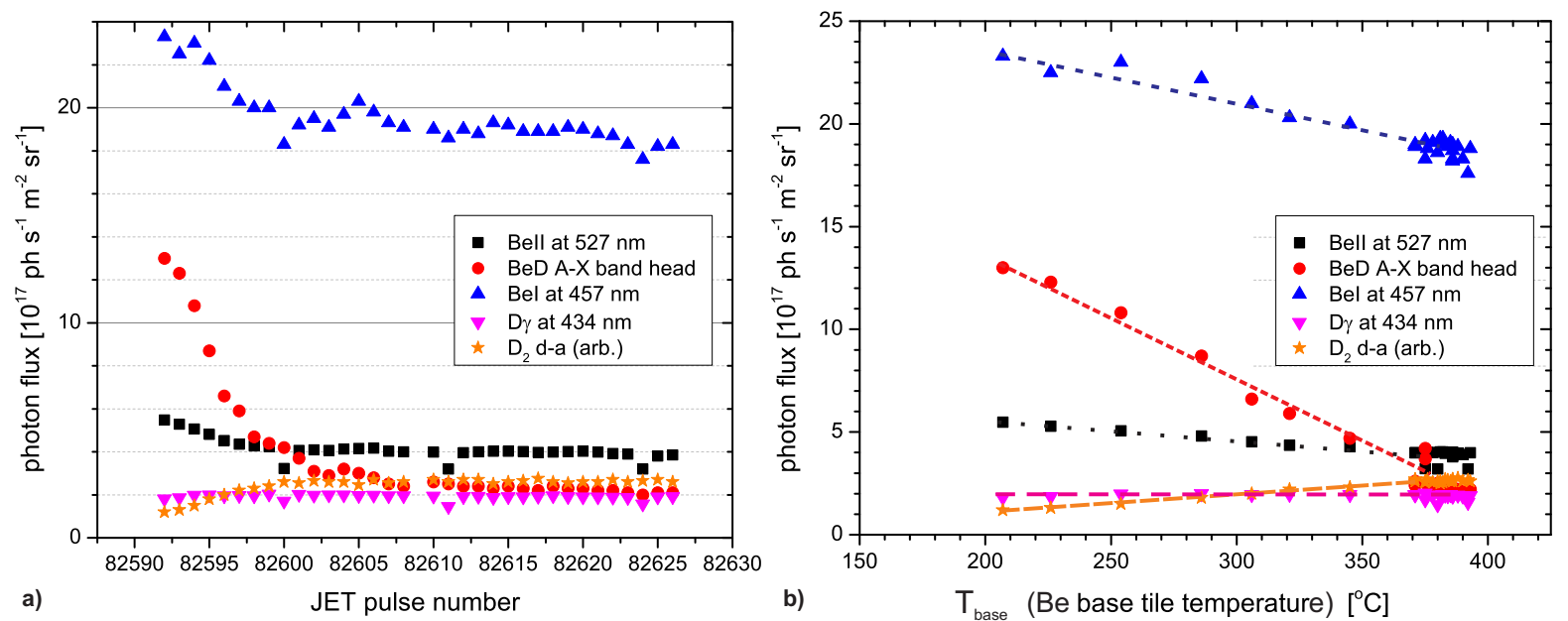

FIG. 6: Experiment (i): a) Discharge to discharge evolution of Be and D photon fluxes with constant plasmas. b)Photon flux evolution of the different species as function of the base temperature of the bulk Be tile $\%$.

The temporal evolution of the brightness of $B e I, B e I I, B e D, D_{2}$, and $D_{\gamma}$ from discharge to discharge at the averaging time window in each discharge at $t=10.0-11.0 \mathrm{~s}$ is shown in fig. 6a). The conversion into actual bulk Be tile temperature ( $T_{\text {base }}$ is depicted correspondingly in fig. 6b) showing variations in the first nine discharges and reaching equilibrium at $T_{\text {base }}=400^{\circ} \mathrm{C}$ with a surface temperature of $T_{\text {surf }} \simeq 520^{\circ} \mathrm{C}$ in the spectroscopic sampling window of the ninth discharge. The residual discharges are within the error bars identical and show the reproducibility of the conditions very well.

The increase of the surface temperature leads to a reduction of all photon fluxes related to Be whereas $D_{\gamma}$ remains constant in all discharges, indicating both constant plasma conditions and identical impinging fluxes to the limiter as full recycling can be assumed. In the same way the $B e D \mathrm{~A}-\mathrm{X}$ band emission decreases, the fraction of observed $D_{2} \mathrm{~d}$-a emission (non-diagonal Fulcher band emission integrated over $580.3-584.1 \mathrm{~nm}$ ) increases indicating a shift in the release mechanism of deuterium from the saturated top surface layers. Fig. 7 shows the emission spectra of the involved atomic lines and molecular bands for the low and high limiter temperature case. Though not the whole ro-vibrational band in both cases is measured, but only a reference wavelength span, the photon fluxes are directly proportional to the particle flux as a) the ro-vibrational population temperature is not varying significantly with the change of the surface temperature and b) the wavelength span covers at least one complete vibrational transition. Detailed studies on the 


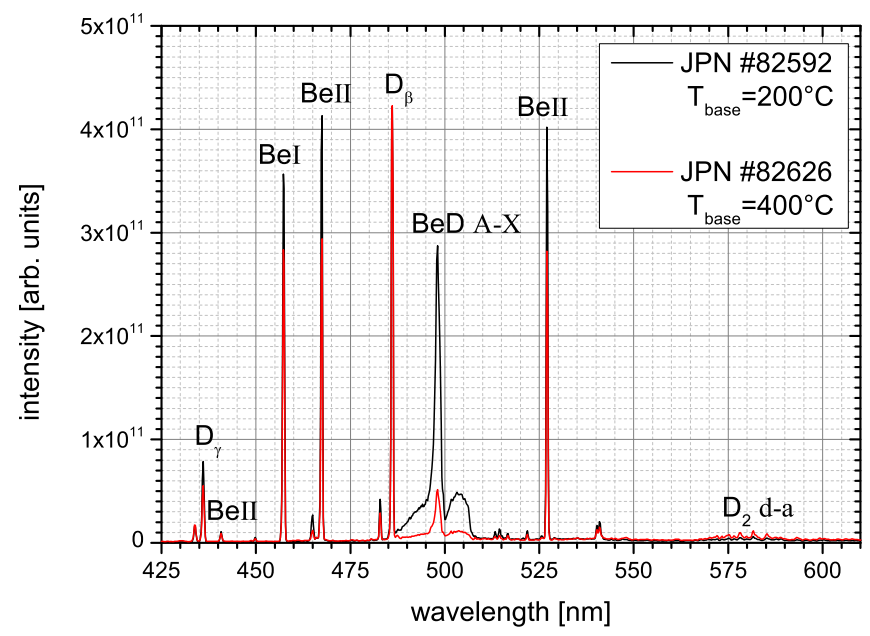

FIG. 7: Emission spectra recorded at the Be limiter at the first and last discharge of the series in experiment I representing low $T_{\text {base }}$ [JPN \#82592] and high $T_{\text {base }}$ [JPN \#82626] conditions.

temperature dependence of the Fulcher- $\alpha$ band were done in limiter plasmas in TEXTOR under similar plasma conditions, but graphite surfaces confirming that the slight increase of the rotational population temperature according to the Boltzmann distribution would be covered [18]. With the previously discussed assumptions, one can conclude that the released deuterium molecular particle flux is doubled in the important discharge series (JPN \#82592 - 82601) in which the the BeD flux dropped down close to the detection level. Under the present high $T_{e}$ conditions at the limiters, the destruction of $\mathrm{D}_{2}$ occurs preferably by electron impact dissociation via the molecular ion $\mathrm{D}_{2}^{+}$, which results in effectively one potential $\mathrm{D}$ atom capable to radiate a Balmer photon with the appropriate photon efficiency [18]. This should have resulted in an increase of the $D_{\gamma}$ radiation as representative for the recycling flux, however, the recycling flux measured by $D_{\gamma}$ is constant. In balance, we can conclude that a similar amount of deuterium atoms must result from the dissociation process of $\mathrm{BeD}$ or $\mathrm{BeD}_{2}$ which starts from the surface. In principle, a full balance would be possible if the complete molecular bands would have been measured and simulated, but this was not accessible with the applied diagnostic equipment.

The line emission resulting from the Be ion (here e.g. BeII at 527nm) is hereby representative for the total Be sputtering source, including bare physical sputtering and chemical assisted physical sputtering, and the band emission of BeD (BeD A-X band head 496.0nm to $499.4 \mathrm{~nm}$ ) is solely representative for the branch of sputtering related to chemical assisted physical sputtering. The line emission originating from the neutral Be atom (here e.g. $B e I$ at $457 \mathrm{~nm}$ ) results from physical sputtering and the fraction of Be sputtering via $\mathrm{BeD}$ which dissociates via $B e D+e \rightarrow B e+D+e^{\prime}$. Thus, the particle flux ratio of $B e I$ to $B e I I$ even provides information on the dissociation chain as any number smaller than unity indicates that not all $\mathrm{BeD}$ delivers finally a Be which can potentially radiate with the corresponding photon efficiency. Moreover, the application of appropriate inverse photon efficiencies, so-called S/XB, for the BeII 527nm line $[15,10]$ and the normalisation to the impinging ion flux, determined by $D_{\gamma}$ and multiplied with the corresponding S/XB-value 
leads to the total Be sputtering yield $Y_{B e}^{t o t}=Y_{B e}^{\text {phys }}+Y_{B e}^{\text {chem }}$.
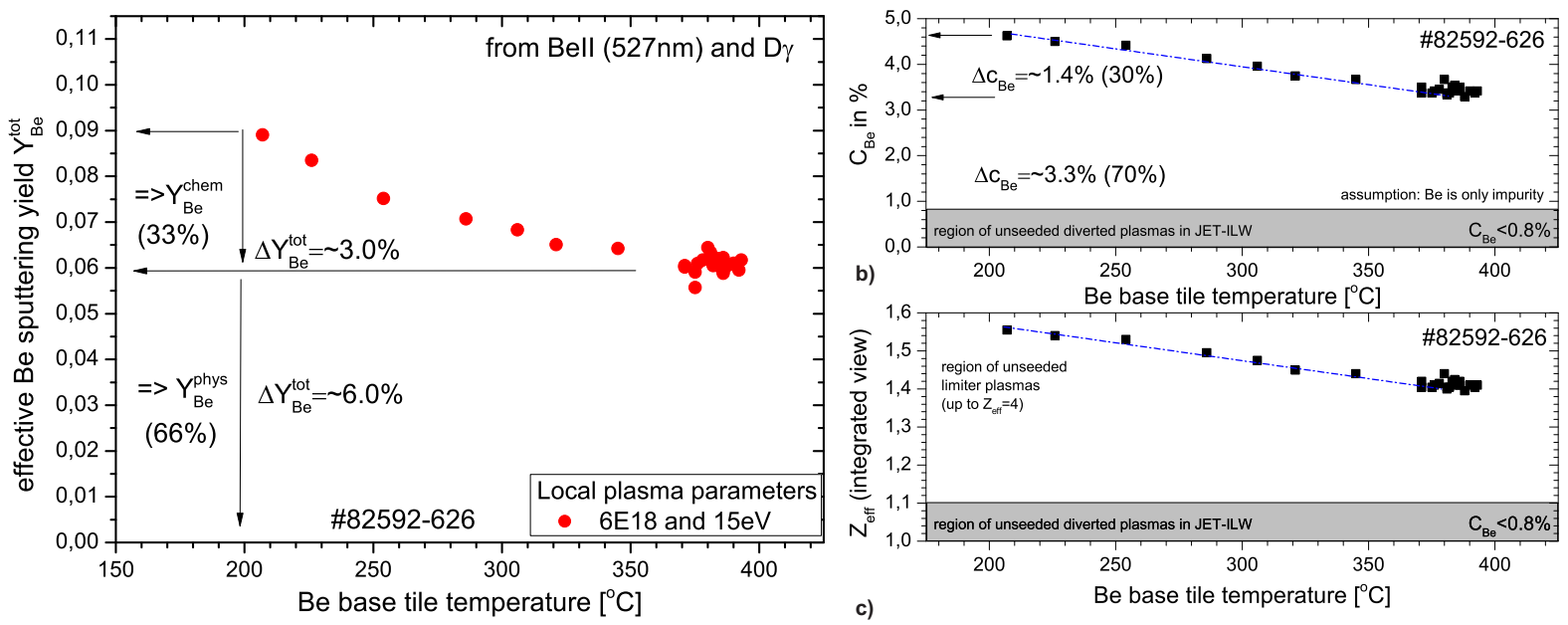

FIG. 8: Experiment (i): a) Contributors to $Y_{B e}^{\text {tot }}$ as function of $T_{\text {base }}$. b) Variation of $c_{B e}$ with $\left.T_{\text {base }} . c\right)$ Variation of $Z_{\text {eff }}$ with $T_{\text {base }}$.

\subsection{The composition of the total Be sputtering yield}

In fig. 8a), $Y_{B e}^{\text {tot }}$ is shown as function of measured temperature of the observed Be tile $T_{\text {base }}$ determined by IR-thermography. It should be again noted that $T_{\text {base }}$ is measured at the actual break-down of the discharge and the incremental peak temperature $\Delta T$ increase by about $100 \mathrm{~K}$ until the measurement window at $t=10-11 \mathrm{~s}$ is reached during the plasma discharge. This is however averaged over the observation spot. A clear linear drop of $Y_{B e}^{t o t}$ with increasing temperature can be seen in the first nine identical plasma discharges till the maximum reachable temperature of the Be tile of about $520^{\circ} \mathrm{C}$ at the sampling time window has been reached. This strong reduction in $Y_{B e}^{t o t}$ by $33 \%$ is caused by the reduction of $Y_{B e}^{\text {chem }}$ which vanishes almost completely at the highest $T_{\text {base }}$ according to the $B e D$ emission described before. Therefore, we can conclude that for the given plasma conditions at the limiter, $T_{e}=15 \mathrm{eV}$ and $n_{e}=6 \times 10^{18} \mathrm{~m}^{-3}$, determined by local $B e I I$ and Balmer-line ratio analysis, about $1 / 3$ of $Y_{B e}^{\text {tot }}$ is originating from $Y_{B e}^{\text {chem }}$ and 2/3 from regular physical sputtering $Y_{B e}^{p h y s}$. This composition is in good agreement with MD modelling predictions [20] for the BeD release at an impact energy of $75 \mathrm{eV}$ which we can assume for this experiment considering $T_{e}=T_{i}$.

From a comparable analysis using $B e I$ at $457 \mathrm{~nm}$ instead of $B e I I$, we obtain a reduction of $Y_{B e}^{\text {tot }}$ by $25 \%$. As the $\mathrm{Be}^{+}$reflects the end product along the dissociation chain, we can conclude that the difference lays in the dissociation path and the BeI to BeII flux ratio reveals that the preferred dissociation path is via the molecule $B e D(75 \%)$ and only about $25 \%$ is following the destruction in plasma via $B e D+e \rightarrow B e D^{+}+e+e^{\prime}$. Note, that a recombination of $\mathrm{Be}$ and $\mathrm{D}$ into the $\mathrm{BeD}$ molecule can be excluded at the high and fully ionising electron temperatures at the limiter contact point and the near SOL of JET.

Further information can be obtained by comparing the reduction of $Y_{B e}^{t o t}$ with the change 
of the core concentration $c_{B e}$ (fig. 8b) deduced from $Z_{\text {eff }}[7,13]$ assuming solely Be a impurity in the plasma (fig. 8c): under otherwise constant plasma conditions, $c_{B e}$ drops in the same manner as $Y_{B e}^{\text {tot }}$ with $T_{\text {base }}$ by about 30\%. Comparing the absolute values, the erosion yield corresponds to twice the concentration in the plasma which suggests an impurity screening of $50 \%$ and that the ratio between gross and net erosion is also a factor 2 as the limiters represent the only source of Be. Comparison between post-mortem analysis and campaign averaged spectroscopy of a comparable Be limiter tile in teh centre of the inner wall limiter confirmed independently a factor two between integral gross and net Be erosion rates during the first year of operation of the JET-ILW [27]. Moreover, the interlink between Be source and Be core content under otherwise constant conditions, in particular identical impinging deuterium ion flux as reflected by the constant $D_{\gamma}$ in fig. 6 , confirms that the chemical assisted physical sputtering of Be represents an additional sputtering channel of Be under the given conditions. However, the measurement of BeII, thus the flux of Be ions, provides still the total flux of eroded Be but the interpretation as bare physical sputtering is incorrect as the chemical assisted physical sputtering provides an additional sputtering channel contributing to $\mathrm{Be}^{+}$. The underlying release mechanism is in both erosion processes similar and based on the energetic bombardment by deuterons which is consistent with observations made in PISCES [12].

A potential impact of a thick co-deposited layer at the interaction location can be excluded. Figure. 9 shows the emission of the $B e D$ A-X band and $D_{\gamma}$ during the short limiter start-up phase in the three discharges prior and post the series of plasmas in experiment I. The local plasma conditions are slightly different as the recycling flux suggests, however, clearly the emission of $\mathrm{BeD}$ is detectable after experiment I. The local temperature of the limiter reflects in these discharges with short interaction time the temperature of the actively heated Be limiters $200^{\circ} \mathrm{C}$ as at the beginning of experiment $\mathrm{I}$. The appearance of the $B e D$ A-X emission is therefore clearly connected to a sputtering

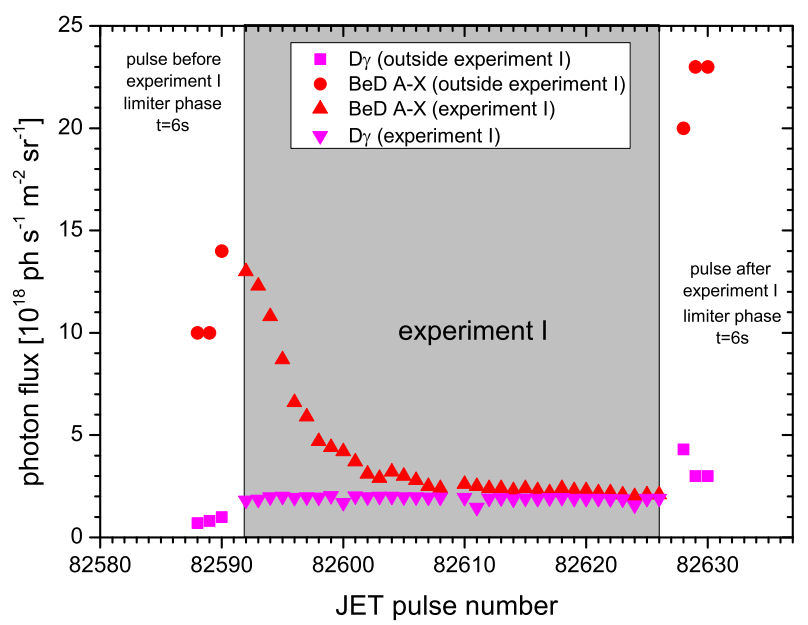

FIG. 9: Appearance of BeD A-X emission in the short limiter start-up phase of three discharges directly prior and post the series of experiment I discharges 
process of bare Be and not a thick co-deposited layer. Moreover, post-mortem analysis of comparable tiles confirm after extraction that the observed limiter tiles reflect net-erosion areas over the whole period of the campaign. This erosion is dominated by the limiter phase or limiter discharges similar to the ones discussed in this paper.

\subsection{Comparison with the composition of the total $\mathrm{C}$ sputtering yield}

The impurity release behaviour is to a certain extent similar to the sputtering of carbon from graphite with observation of $\mathrm{C}^{+}(C I I$ emission $)$, representing physical and chemical erosion, and CD (CD A-X band emission), reflecting solely the chemical erosion with predominantly methane release and break-up into CD [19]. Also the chemical sputtering of graphite vanishes at higher surface temperatures as measured in TEXTOR under comparable edge plasma conditions as in JET [19] and analytically described in [23]. However, the chemical sputtering process and the energy of the radicals produced is different in the case of Be and $\mathrm{C}$, whereas the latter is thermally released. Moreover, at the low energy range, an energy threshold for Be sputtering of about $10 \mathrm{eV}$ has been calculated [20] which inhibits erosion by low energetic ions or even thermal atoms as it occurs in the case of C. Note that this fact has vital importance on the material migration behaviour in the JET-ILW as the Be erosion in the far-SOL, thus, the main chamber wall and the divertor, will be reduced in diverted magnetic configurations in comparison with JET-C. This can be already seen in the dramatic reduction of the primary $\mathrm{C}$ source in comparison with the Be in JET-ILW [5] as well as the initial post-mortem analysis studies of limiters and divertor plasma-facing components [21].

\section{Total Be sputtering yield as function of $E_{i n}$}

Experiment (II) aims to determine the dependency of $Y_{B e}^{t o t}$ on $E_{i n}$ in the accessible range of limiter plasma conditions with the JET-ILW. As no direct measurement of the impact energy exists, we still assume the validity of $E_{i n} \simeq 5 \times T_{e}$ over the full plasma parameter range. Fig. 10 shows the measured total sputtering yield $Y_{B e}^{t o t}$ as function of the local $T_{e}$ deduced from local spectroscopy discussed before. The measured $T_{e}$ in the SOL varies between $5 \mathrm{eV}$ and $35 \mathrm{eV}$ and is inversely proportional to the central plasma density which has been varied in a controlled manner by deuterium fuelling ramps. $Y_{B e}^{\text {tot }}$ increases moderately with impact energy from $T_{e} \simeq 5 \mathrm{eV}$ up to about $T_{e} \simeq 30 \mathrm{eV}$ which is in line with the increase of the physical sputtering process by deuterons. We observe the dominance of self-sputtering by impinging Be ions at $T_{e}>30 \mathrm{eV}$ and, thus, $E_{i n}>150 \mathrm{eV}$ which compromises the definition of the yield and normalisation to the deuterium ion flux. The contribution of Be self-sputtering to the total sputtering decrease with lower electron temperature and higher electron density. It is in the normal operational window of JETILW baseline discharges with phases of magnetic limiter configuration (start-up, limiter flat-top, and ramp-down) and pure ohmic heating usually not of importance $\left(Z_{\text {eff }}<1.8\right)$ in comparison with the sputtering contribution determined by the impinging deuterons. Only in dedicated experiments like here described, the full operational window is explored 


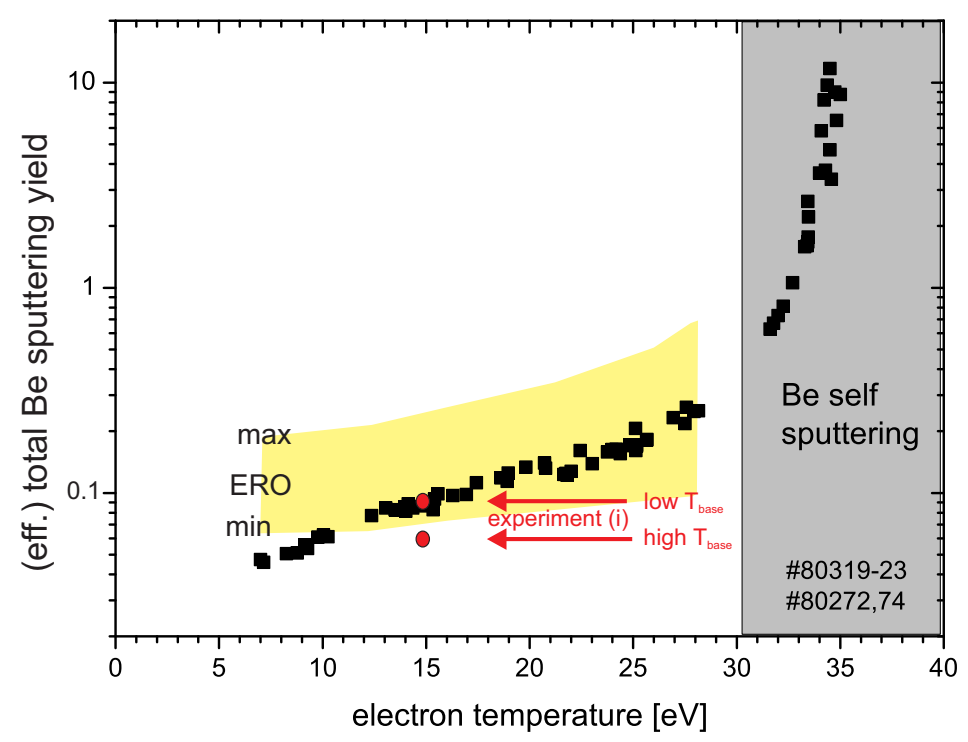

FIG. 10: $Y_{B e}^{\text {tot }}$ as function of the local $T_{e}$ or $\frac{1}{5} E_{\text {in }}$ in experiment (ii). The shadowed area indicated ERO modelling with the compiled sputtering data $E R O_{\max }$ and $E R O_{\min }$ for the bare physical sputtering yields [13].

with on purpose high electron temperatures to study in particular the sputtering yield or the power load to the limiters. At the lowest controlled accessible $T_{e} \simeq 5 \mathrm{eV}$ in limiter configuration before MARFE formation $\left(T_{e} \simeq 1 \mathrm{eV}\right)$ and inner wall detachment occurs, still the corresponding impact energy of $E_{\text {in }} \simeq 25 \mathrm{eV}$ is above the energetic threshold for sputtering. Thus, there is no access to verify experimentally the energetic threshold predicted by MD in the first place. Moreover, there is no direct access to conditions comparable to diverted plasma conditions with large distance between the Be wall cladding and the separatrix where the residual deuterium plasma flux to recessed wall represents a strong contributor of the primary Be source at low $T_{e}$ and $E_{i n}$ in addition to energetic charge exchange neutrals which cause Be erosion.

The measurements at lowest and highest $T_{\text {base }}$ from experiment (I) are marked in fig. 10; indicating the impact of $Y_{B e}^{\text {chem }}$ at one particular $T_{e}$ value. The measurement at cold limiter conditions at $E_{i n}=75 \mathrm{eV}$ fits excellent in the point band of experiment (II). The measurement point at highest limiter temperature in experiment (I), thus, without chemical assisted physical sputtering is well below the measurements of experiment (II). This indicates the importance of the second sputtering channel in the overall Be erosion yield. The yellow marked area spans the set of ERO calculations of the Be erosion yield simulating the effective sputtering Be erosion yield for a synthetic spectroscopic diagnostic considering the same line-of-sight and plasma conditions as present in experiment II. The calculations are performed solely under the assumption of bare physical sputtering of Be by deuterium and Be self-sputtering. The upper boundary is given by the so-called ERO-max envelope of available experimental and calculated sputtering yields as input parameter as described in [13]. Correspondingly, the lower boundary is determined by the most optimistic set of available sputtering yield values. In order to achieve the match depicted in fig. 10, the impinging ion flux, benchmarked by the measured $D_{\gamma}$ photon flux, 
had to be reduced by a factor 2. In other words, the effective erosion yield in ERO for the most optimistic case (ERO-min) in fig. 10 would be de-facto a factor two larger and everywhere clear above the experimental data if the measured ion flux would have been applied. Though a $50 \%$ surface coverage of the Be surface with deuterium was assumed in the ERO calculations, the process of chemical assisted physical sputtering was not yet included in the suite of atomic and molecular processes. Consideration of the process would increase further the discrepancy between modelling and experiment as it is indicated by the larger gap between the lowest envelope of ERO-min at $T_{e}=15 \mathrm{eV}$ for the case of the almost bare physical sputtering measured at the high $T_{b a s e}$ of experiment I. In other words, even with the additional measured sputtering channel, the corrected ERO-min calculations simulating the experiment overestimate by about a factor 2.3. The discrepancy increases further at the low energy range where the corrected ERO-min lays outside of the measurements without consideration of a potential higher fraction of $\mathrm{BeD}$ contributing to the effective sputtering yield. The match is better with increasing importance of Be self sputtering. This indicates that the set of Be sputtering yields used in ERO-min at the lower energy range needs to be critically revisited. Additional benchmarks including the process of chemical assisted physical sputtering employing MD calculated Be sputtering yields by [20] are foreseen to benchmark the code further with JET-ILW experimental results.

The current experimental results provide only a single measurement of the composition of $Y_{B e}^{t o t}$ for the impinging energy of $E_{i n}=75 \mathrm{eV}$ as the experiment is costly in experimental time and relies on high repetition rate of discharges in JET-ILW to heat-up the limiter by plasma impact. It is expected that the composition of the contributors to $Y_{B e}^{t o t}$ will likely change [20] in the plasma parameter range covered in experiment II with a higher fraction of $Y_{B e}^{c h e m}$ at lowest energies, representing the fist wall conditions in divertor plasmas, and negligible contribution at the high energetic range, representing low density conditions in plasma start-ups for advanced plasma scenarios. Apart from the energy dependence which favours bare physical sputtering at high impact energies and deeper penetrations of deuterons into the Be matrix, also the reservoir of available deuterium impinging the limiter surface reduces on cost of additional Be impinging flux. This self-amplified process is usually inhibited by sufficient high deuterium fuelling in JET discharges to protect the first wall from too high Be concentrations in the plasma which can subsequently cause $\mathrm{W}$ sputtering when the divertor coils are switched on and the significant flux of plasma and impurities are hitting the tungsten divertor target plates.

Finally, we need to stress that the discussed Be sputtering yields represent effective yields due to averaging over the observation area on the 3D-geometry of the Be limiter tile. Averaging of local plasma conditions $\left(T_{e}, n_{e}, E_{i n}\right)$, impact angles, and impinging fluxes within the observation chord takes place. However, the here discussed measurements provide a good data set to new benchmarks with the ERO code, including the chemical assisted Be sputtering. ERO treats the involved processes on the atomistic level and can disentangle the individual processes in order to make predictions in more complex geometries and conditions like in ITER. 


\section{Discussion}

The main information obtained in these experimental work can be summarised as follows: (i) the effective sputtering yield of Be as function of impact energy $\left(E_{\text {in }}=25-175 \mathrm{eV}\right)$ and surface temperature $\left(T_{\text {surf }}=200^{\circ} \mathrm{C}-520^{\circ} \mathrm{C}\right)$ has been determined in limiter configuration in-situ by emission spectroscopy in JET. (ii) At a medium impact energy $\left(E_{\text {in }}=75 \mathrm{eV}\right.$ ) two contributors to the total effective Be sputtering yield were identified. Two third of the eroded Be originates from bare physical sputtering and one third from chemical assisted physical sputtering which practically vanishes at high Be limiter temperatures $\left(T_{\text {surf }}>520^{\circ} \mathrm{C}\right)$. (iii) Be self sputtering dominates the erosion at high impact energies $\left(E_{\text {in }}>150 \mathrm{eV}\right)$ and causes effective yields far beyond 1 whereas the normalisation to the impinging ion flux to the limiter is no longer useful. (iv) The effective gross Be sputtering yield drops to extremely low values, below $4.5 \%$, at the accessible lowest impact energy $\left(E_{\text {in }} \simeq 25 \mathrm{eV}\right)$ achievable in limiter configuration in JET. (v) The corresponding lowest effective net Be sputtering yield amounts $2.3 \%$ under consideration of the observed relation (factor 2) between net and gross Be erosion at the centre limiter tile by post-mortem analysis and optical spectroscopy.

\subsection{The role of chemical assisted physical sputtering in the over- all Be erosion}

Though no dedicated experiments at lower impact energies in JET exist, it is expected that the contribution of chemical assisted physical sputtering gains further importance as the emission of $B e D$ from recessed main chamber wall cladding tiles in divertor configuration suggests. Indeed, both MD modelling [18] and experiments at PISCES-B [12] predict at impact energies $E_{i n}<50 \mathrm{eV}$, the dominance of the Be chemical assisted physical sputtering over the bare physical sputtering before an energetic threshold at about $E_{\text {in }}<10 \mathrm{eV}$ inhibits further sputtering. The measured JET-ILW value for the amount of Be sputtered as $\mathrm{BeD}$ is depicted in fig. 11 and shows good agreement with both MD modelling and the experimental PISCES-B data. The PISCES-B measurements suggest that sputtering at higher energies is mainly unaffected by chemical assisted physical sputtering and only bare physical sputtering is the origin of eroded Be. This corresponds at JET to the region where experimentally self sputtering starts to become the dominant process. Contrary, at low impact energies the release as molecular compound is dominant over the release as energetic Be atom by bare physical sputtering. However, the experiments, both in JET and PISCES-B, cannot exclude that the chemical assisted physical sputtering takes place with the release of the stable molecule $\mathrm{BeD}_{2}$ rather than the radical $\mathrm{BeD}$. There is no direct spectroscopic access to $\mathrm{BeD}_{2}$ in the experiments.

The contribution of the molecular compounds of Be to the total sputtering yield of $\mathrm{Be}$ depends on the amount and form of $\mathrm{D}$ embedded in the Be surface layers within the ion implantation range. Without sufficient deuterium reservoir present, the release of chemical compounds by physical impact is much reduced which means independently of the destruction process of a BeD molecule, at higher surface temperatures and lower fuel content in the Be, less molecular-related sputtering can be expected. Here, the high impinging deuterium ion flux in limiter configuration, a few $10^{21} D s^{-1} \mathrm{~m}^{-2}$ and corresponding 


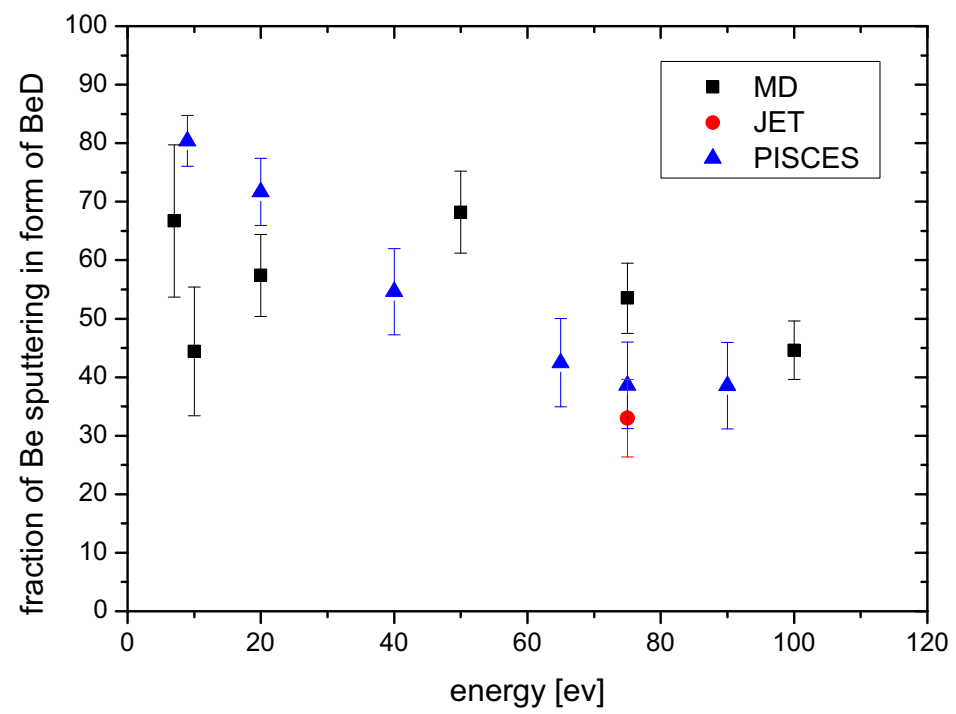

FIG. 11: Fraction of total Be sputtering released as BeD in JET, PISCES [12] and MD calculations as function of impact energies of deuterons. The variation in the low energy end of the MD calculations is caused by low statistics in the overall small sputtering yield.

fluence per inboard limiter phase $(\delta t=12 s)$ of about $3 \cdot 10^{22} \mathrm{Dm}^{-2}$, ensures a quasi "saturation" of the surface by deuterium and provides the necessary reservoir of $\mathrm{D}$ in the Be matrix to enable the above described chemically assisted physical sputtering. Present ERO modelling assumes a constant deuterium content of $50 \%$ in the thickness of the interaction matrix used in the code. This is a simplification to the really complex situation with regard to the pulsed operation of JET.

\subsection{The role of the deuterium content in Be on the sputtering}

Indeed experiment I, used to determine the composition of the sputtered Be, represents a complex process sequence of (i) high flux deuterium loading of Be, (ii) implantation of $\mathrm{D}$ in Be in different vacancies and trap sites. In parallel, (iii) the Be limiter temperature increases during the deuterium loading varying the populated vacancies and traps, and (iv) outgassing during the discharge (e.g. in fig. 2 at $t=17 \mathrm{~s}$, when the limiter contact point changes from inboard to outboard and no deuterium gas is injected, but the density rises) takes place as well as (v) post-discharge outgassing at elevated temperature. In fact the ratch-up of the $T_{\text {base }}$ from one discharge to the next discharge represents a poor way of thermal desorption with stepwise increase of the desorption temperature at almost constant hold times for about $20 \mathrm{~min}$ equivalent to the time between two discharges. In parallel to this temporal variation of the deuterium content in the Be PFCs, which takes place intra- and inter-discharge, the observed Be sputtering via physical and chemical assisted physical sputtering takes place. As discussed before in sec. 3, the sputtering via $\mathrm{BeD}$ and associated production of $\mathrm{D}$ out of the molecular dissociation, and the increase of $D_{2}$ desorption is anti-reciprocal until almost all $\mathrm{BeD}$ is vanished. Thus, with respect 
to the amount of deuterium released by thermal desorption or as deuterium break-up product of chemical assisted physical sputtering, both processes are interconnected to the amount of $\mathrm{D}$ in the Be matrix - populated in vacancies and traps. The reference amount of D measured spectroscopically by Balmer radiation at the limiter is almost constant for all discharges, indicating that the net amount of released deuterium remains constant in this series of discharges.

Comparison with laboratory experiments dealing with deuterium retention and release in Be is only qualitatively possible due to the large difference in the impinging flux $\left(\simeq 10^{17} \mathrm{Ds}^{-1} \mathrm{~m}^{-2}\right)$ and fluence $\left(\simeq 10^{22} \mathrm{Dm}^{-2}\right)$ in comparison to JET conditions described before including the complex loading and outgassing. In [22], dedicated studies at (a) low surface temperature $\left(T_{\text {surf }}=100^{\circ} \mathrm{C}\right)$, below the starting temperature of the Be tile observed here at JET, and at (b) high surface temperature $\left(T_{\text {surf }}=280^{\circ} \mathrm{C}\right)$ were carried out. Dedicated thermal desorption revealed in case (a) six trap sites, the two low energetic ones are related to supersaturation which vanishes with temperature as demonstrated in case (b). Two other trap sites at about $\left(T_{\text {surf }}=430^{\circ} \mathrm{C}\right)$ are only populated significantly at higher loading temperatures and have been attributed to an amorphous state which can induce direct release of $\mathrm{BeD}$ or $\mathrm{BeD}_{2}$ till full thermal decomposition of the molecular species sets in [12], leaving a very narrow temperature window for the molecular release via $\mathrm{BeD}$ or $\mathrm{BeD}_{2}$. Though the conditions in the laboratory studies and the tokamak experiment are different, we can exclude that the observed spectroscopic $B e D$ emission in JET is attributed to a thermal desorption process via the volatile molecule $B e D_{2}$ and subsequent dissociation into $B e D$. $B e D$ is most prominent at the lowest surface temperatures at JET and almost absent at the temperature window where the release according to the laboratory results shall be strongest. Therefore, we conclude that chemical assisted physical sputtering is the origin of the observed $B e D$ emission and the increased sputtering yield with respect to ordinary Be sputtering as it can be calculated by TRIM for impact energies as present in the limiter plasmas.

\subsection{Comparison with TRIM calculations and laboratory exper- iments}

In zeroth order it is possible to compare the absolute values of the effective Be sputtering yields determined in JET with calculations from binary-collision approximations (BCA) like TRIM [11] or with recent experimental results from the linear plasma device PISCES [25]. Considering the conditions of experiment I, TRIM calculations predict a net erosion yield of about $2.8 \%$ at an impinging deuteron energy of about $E_{\text {in }} \simeq 75 \mathrm{eV}$ under perpendicular impact on a bare Be surface: simple physical sputtering of $B e$ by $D^{+}$. In JET, we measured by spectroscopy an effective Be gross erosion yield of about 9.0\% which includes both erosion processes: chemically assisted physical sputtering and physical sputtering. If we only consider the pure physical sputtering part - as it is in TRIM - we must reduce the effective total erosion yield $Y_{B e}^{\text {tot }}$ by the contribution $Y_{B e}^{\text {chem }}$ which results in $Y_{B e}^{\text {phys }}=6.0 \%$ equivalent to a reduction of $Y_{B e}^{\text {tot }}$ by about one third at the given impact energy $\left(E_{\text {in }}=75 \mathrm{eV}\right)$. Furthermore, TRIM considers only the net erosion, thus, it is required to include in the JET results the ratio between net and gross erosion 
which is approximately a factor two as shown before. This results in an effective net erosion yield $Y_{B e}^{\text {phys }}=3.0 \%$ which is on a first glance comparable to the simple TRIM prediction. However, the effective Be erosion yield deduced in JET is still representing a strongly averaged quantity: (a) no single impact energy, but a distribution, (b) no single surface temperature, but an averaged value, (c) no perpendicular impact, but under an averaged impact angle of about ${ }^{6} 0^{\circ}$, (d) not a homogenous impinging deuteron flux, but a three dimensional distribution due to the limiter geometry, (e) no pure deuteron sputtering, but Be self-sputtering can contribute to the total yield etc. Moreover, the Be PFCs in JET are not representing a pure and clean Be matrix as in TRIM, but certainly a high fraction of fuel is embedded in the interaction zone as shown in the previous section which impacts on the sputtering yield. In order to include all this averaging and the impact of the additional processes, which can either increase the bare yield (e.g. (c) causes a factor 10 increase [13]) or decrease (e.g. (d) can cause higher prompt redeposition), in the interpretation of the experimental results, it is of advantage to compare the experimental results with dedicated modelling to disentangle the different contributions to the sputtering. The ERO code is capable to include most of these processes, but needs to be further improved (e.g. inclusion of chemical assisted physical sputtering) and verified by tokamak experiments in order to increase the confidence in ITER predictions.

Even in a linear plasma in simple cylindrical geometry like in PISCES is the comparison with BCA calculations not straight forward as shown in [25] where a discrepancy of a factor 5 to 10 to TRIM calculations was observed, i.e. a lower erosion yield than expected. In addition to common processes between tokamak plasmas and linear device plasmas such as the impact of re-deposition, chemical assisted physical sputtering or fuel content on the Be sputtering yield, also linear-plasma-specific effects play a role in the measured sputtering yields. Two effects were described in [25]: the distribution of impinging ionic particles $\left(D+, D^{2+}, D^{3+}\right.$ composition) as well as the modification of the surface morphology. The later one, which seems to be responsible for a factor 2-3 reduction in the Be erosion yield measured in PISCES, has not been observed under tokamak conditions, probably due to averaging effects described before, and doesn't need to be considered. The complex impinging deuterium ion distribution is also not of importance in the far-SOL of a tokamak where only $D^{+}$needs to be considered. Associated modelling is required to explain the residual reduction of the sputtering yield with respect to TRIM.

\subsection{Conclusions for ITER predictions}

The current experiments at JET give only limited access to different impinging flux or surface temperature conditions, but it provides overall the closest conditions with respect to ITER and can be used for direct ITER predictions with respect to erosion yields and for code benchmarking. Considering the factor 2.5 larger erosion found in the most optimistic set of input atomic and surface data $\left(\mathrm{ERO}_{\min }\right.$ in ERO simulations of the JET-ILW experiments), the lifetime of the first wall in ITER is longer by the same factor than current published predictions [3]. Chemical assisted physical sputtering in ERO has not yet been considered, but the bare BeII emission of different transitions has been used as critical benchmark with JET. Therefore, further detailed studies with variation of a single parameter, i.e change of the Be surface temperature, the impact energy of deuterons down to the predicted threshold energy, and the impinging flux, would be required to further 
improve the modelling input and the ITER predictions.

These detailed studies with variation of a single controlled parameter can only be performed in linear plasma devices such as PISCES and in laboratory experiments within their validity range in flux and fluence. Therefore, a combined approach of laboratory experiments, JET experiments and associated verified MD and ERO calculations will finally lead to better predictions of the erosion-dominated lifetime of the ITER Be first wall with its complex three dimensional shaping. 
This work was carried out within the framework of EFDA, the Forschungszentrum Jülich and the US DOE Contract: DE-FG02-07ER54912. The views and opinions expressed herein do not necessarily reflect those of the European Commission.

\section{References}

[1] V. Philipps et al.; Fus. Eng. Des. 85 (2010) 1581-1586

[2] S. Carpentier et al., J. Nucl. Mater. 415 (2011) S165

[3] D. Borodin et al., Phys. Scripta T145 (2011) 14008

[4] G.F. Matthews et al., J. Nucl. Mater. 438 (2013) S2

[5] S. Brezinsek et al., J. Nucl. Mater. 438 (2013) S303

[6] S. Brezinsek et al., Nucl. Fus. 53 (2013) 083023

[7] J.W. Coenen et al., Nucl. Fus. 53 (2013) 073043

[8] G.F. Matthews et al., Phys. Scripta T145 (2011) 014001

[9] G. Duxbury et al., Plasma Phys. Control Fusion 40 (1998) 361

[10] S. Brezinsek et al., 41st EPS on Plasma Physics, ECA 37D (2013)

[11] W. Eckstein et al., Report IPP 9/132 (2002)

[12] D. Nishijima et al., Plasma Phys. Control Fusion 50 (2008) 125007

[13] D. Borodin et al., J. Nucl. Mater. 438 (2013) S267

[14] R.P. Doerner et al., J. Nucl. Mater.390-391 (2009) 681

[15] ADAS, http://adas.phys.strath.ac.uk

[16] P.C. Stangeby, The plasma boundary of magnetic fusion devices, IOP publishing (2000)

[17] G. Arnoux et al., Nucl. Fus. 53(2013) 073016

[18] S. Brezinsek et al., Plasma Phys. Control Fusion 47 (2005) 615

[19] S. Brezinsek et al., J. Nucl. Mater. 363-365 (2007) 1119

[20] C. Björkas et al., New J. Physics 11 (2009) 123017

[21] A. Widdowson et al., Phys. Scripta T159 (2014) 014010

[22] M. Reinelt et al., New J. Physics 9 (2009) 043023 
[23] J. Roth et al., Nucl. Fus. 361647

[24] A. Kreter et al., Plasma Phys. Control Fusion 47 (2003) 199

[25] R.P. Doerner et al., J. Nucl. Mater. 438 (2013) S272

[26] G. Arnoux et al., Phys. Scripta T159 (2014) 014009

[27] S. Brezinsek et al., IAEA 21st Fusion Energy Conference 2014, EX/P5-26 